\title{
Inflammation induced by influenza virus impairs human innate immune control of pneumococcus
}

\author{
Simon P. Jochems ${ }^{1,8 \star}$, Fernando Marcon ${ }^{2,8}$, Beatriz F. Carniel ${ }^{1}$, Mark Holloway ${ }^{1}$, Elena Mitsi ${ }^{1}$, \\ Emma Smith', Jenna F. Gritzfeld', Carla Solórzano', Jesús Reiné1, Sherin Pojar', Elissavet Nikolaou', \\ EstherL.German', AngieHyder-Wright ${ }^{1,3}$, Helen Hill1,3, Caz Hales ${ }^{10}{ }^{1,3}$, Wouter A. A.deSteenhuijsen Piters ${ }^{4,5,6}$, \\ Debby Bogaert ${ }^{4,5,6}$, Hugh Adler (1) ', Seher Zaidi', Victoria Connor ${ }^{1,3}$, Stephen B. Gordon 1,7, Jamie Rylance (D) , \\ Helder I. Nakaya ${ }^{2 \star}$ and Daniela M. Ferreira ${ }^{1 \star}{ }^{1 \star}$
}

Colonization of the upper respiratory tract by pneumococcus is important both as a determinant of disease and for transmission into the population. The immunological mechanisms that contain pneumococcus during colonization are well studied in mice but remain unclear in humans. Loss of this control of pneumococcus following infection with influenza virus is associated with secondary bacterial pneumonia. We used a human challenge model with type 6B pneumococcus to show that acquisition of pneumococcus induced early degranulation of resident neutrophils and recruitment of monocytes to the nose. Monocyte function was associated with the clearance of pneumococcus. Prior nasal infection with live attenuated influenza virus induced inflammation, impaired innate immune function and altered genome-wide nasal gene responses to the carriage of pneumococcus. Levels of the cytokine CXCL10, promoted by viral infection, at the time pneumococcus was encountered were positively associated with bacterial load.

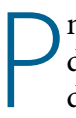
neumonia is a major global health problem; it kills more children under 5 years of age than any other disease ${ }^{1}$. The burden of disease is aggravated by old age, chronic lung disease, immunosuppression and viral co-infection. Secondary pneumonia following pandemic and seasonal influenza virus infection is a significant cause of mortality worldwide ${ }^{2}$.

Nasopharyngeal colonization by Streptococcus pneumoniae (pneumococcus) is common, with $40-95 \%$ of infants and $10-25 \%$ of adults colonized at any given time ${ }^{3}$. Such pneumococcal carriage is important as the prerequisite of infection ${ }^{4}$, the primary reservoir for transmission ${ }^{5}$ and the predominant source of immunizing exposure and immunological boosting in both children and adults ${ }^{6,7}$.

Immune dysregulation caused by infection with respiratory viruses such as influenza leads to increased carriage load ${ }^{8}$. Increased carriage load has been associated with pneumonia incidence and severity, as well as with within-household $S$. pneumoniae transmis$\operatorname{sion}^{5,9-11}$. The mechanisms and markers associated with this pathogen synergy have been difficult to study in human subjects due to the rapidly progressing nature of the disease.

One safe way to simulate influenza infection in the nose is using live attenuated influenza vaccine (LAIV), consisting of cold-adapted influenza viruses. LAIV has been shown to affect subsequent susceptibility to $S$. pneumoniae and to lead to increased carriage load in mouse models of infection and in vaccinated children ${ }^{12,13}$. Furthermore, LAIV administration prior to S. pneumoniae challenge led to a $50 \%$ increase in $S$. pneumoniae acquisition, as detected by molecular methods, as well as a tenfold increase in nasopharyngeal bacterial load ${ }^{14}$.
In mouse models of pneumococcal carriage, recruitment of neutrophils and monocytes to the nasopharynx dependent on the $\mathrm{T}_{\mathrm{H}} 17$ subset of helper $\mathrm{T}$ cells mediates immunological control and clearance ${ }^{15-17}$. Influenza virus infection promotes type I interferons, which interfere with recruitment of these phagocytes, although interferon- $\gamma$ (IFN- $\gamma$ ) is postulated to impair phagocytosis by macrophages through downregulation of the scavenger receptor MARCO (macrophage receptor with collagenous structure) ${ }^{18-20}$. However, the precise immune mechanisms and gene regulators involved in the control and clearance of pneumococcal carriage in humans have not been revealed ${ }^{21}$. Moreover, how these mechanisms are altered during human influenza virus infection remains largely unknown.

Systems-biology approaches have allowed the identification of immune mechanisms associated with protection from infectious diseases and with robust immune responses during vaccination ${ }^{22-28}$. Here, we applied systems biology to nasal samples collected in the setting of human challenge with LAIV and S. pneumoniae, to emulate nasal effects of influenza infection on $S$. pneumoniae carriage. We identified the key cellular mechanisms that control newly acquired pneumococcal carriage in humans, and how they are disrupted following nasal influenza infection.

\section{Results}

LAIV-induced inflammation leads to increased pneumococcal carriage load and acquisition. In a double-blinded controlled randomized clinical trial, we administered LAIV $(n=55) 3$ days prior to $S$. pneumoniae inoculation (day 0 ). To verify the requisite topical

'Department of Clinical Sciences, Liverpool School of Tropical Medicine, Liverpool, UK. ${ }^{2}$ Department of Clinical and Toxicological Analyses, School of Pharmaceutical Sciences, University of São Paulo, São Paolo, Brazil. ${ }^{3}$ Royal Liverpool and Broadgreen University Hospital, Liverpool, UK. ${ }^{4}$ Centre for Inflammation Research, University of Edinburgh, Edinburgh, UK. ${ }^{5}$ Department of Paediatric Immunology and Infectious Diseases, University Medical Center Utrecht, Utrecht, The Netherlands. ${ }^{6}$ Department of Medical Microbiology, University Medical Center Utrecht, Utrecht, The Netherlands. ${ }^{7}$ Malawi-Liverpool-Wellcome Trust Clinical Research Programme, Blantyre, Malawi. ${ }^{8}$ These authors contributed equally: Simon P. Jochems and Fernando Marcon *e-mail: simon.jochems@lstmed.ac.uk; hnakaya@usp.br; daniela.ferreira@lstmed.ac.uk 

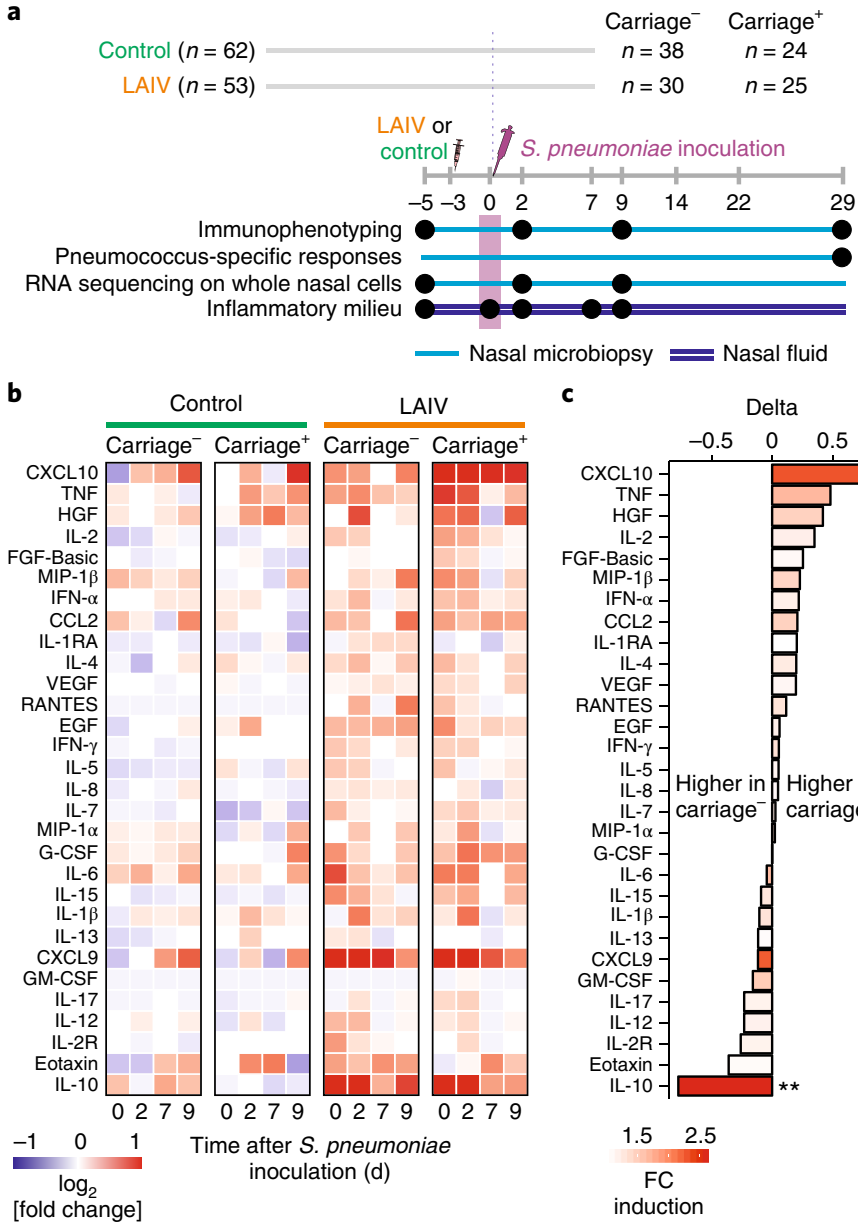

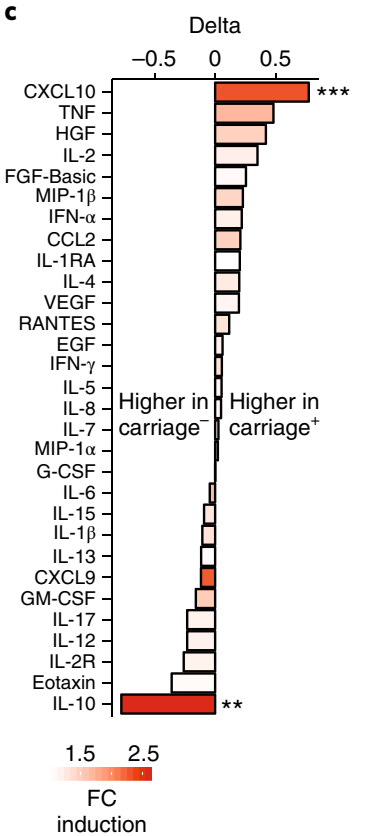

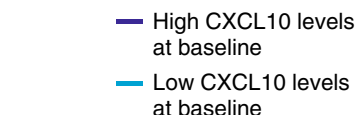

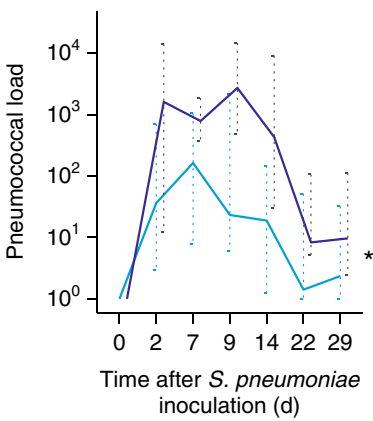

- Virus-positive

- Virus-negative

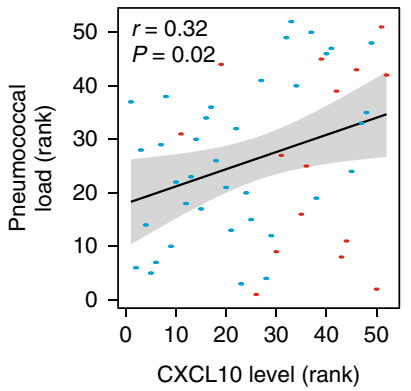

Fig. 1 | LAIV-pneumococcus co-infection leads to excessive proinflammatory responses that are associated with increased pneumococcal load and impaired monocyte recruitment. a, Experimental design of the study. Analyzed time points are indicated by black circles. $\mathbf{b}$, Heat map showing for each cytokine the median $\log _{2}$ [fold change] compared with baseline for the time points $0,2,7$ and 9 days; $n=19$ per group. $\mathbf{c}$, The delta in median log 2 [fold change] following LAIV vaccination just prior to inoculation with $S$. pneumoniae for subjects becoming carriage ${ }^{+}$or carriage ${ }^{-}$(excluding subjects becoming positive by PCR only, who resemble subjects that become carriage ${ }^{+}$by culture as well). The color of each bar represents the median induction in the entire LAIV group. ${ }^{\star \star} P=0.0097$ by two-tailed Wilcoxon test for LAIV carriage ${ }^{-}$subjects comparing IL-10 day 0 with baseline; $P=0.073$ for the LAIV carriage ${ }^{+}$ group. ${ }^{\star \star} P=0.0008$ by two-tailed Wilcoxon test for LAIV carriage+ subjects comparing CXCL10 day 0 with baseline; $P=0.051$ for the LAIV carriage ${ }^{-}$ group. d, Pneumococcal load (median and interquartile range of CFU ml ${ }^{-1}$ in nasal wash shown) for all carriage ${ }^{+}$subjects with high (top quartile, $n=9$ ) or low (all subjects below top quartile, $n=28$ ) CXCL10 concentrations at day 0. $P=0.019$ by two-tailed Mann-Whitney test of area under the curve of logtransformed load over time. e, Scatter plot showing correlation of CXCL10 concentration at baseline with S. pneumoniae load for a second validation cohort $(n=52)$ with an asymptomatic upper respiratory tract virus infection $(n=15)$ or not. Spearman correlation test results and linear regression line with $95 \%$ confidence interval (gray shading) are shown.

application for an effect on pneumococcal carriage, we administered tetravalent inactivated influenza vaccine as a control $(n=62)$. LAIV infection led to transiently increased pneumococcal acquisition at day $2(60.0 \%$ and $40.3 \%$, as detected by molecular methods, in LAIV groups and control groups, respectively) ${ }^{14}$. LAIV also increased S. pneumoniae carriage load in the first 14 days following pneumococcal inoculation ${ }^{14}$ (Supplementary Fig. 1). We collected a series of nasal microbiopsies and nasal lining fluid throughout the study to assess ongoing cellular and cytokine responses. Participants were grouped into those who did not become colonized following S. pneumoniae challenge (carriage ${ }^{-}$) and those who did $\left(\right.$carriage $\left.^{+}\right)$, as determined by classical microbiology (Fig. 1a). To investigate whether LAIV-induced immune responses were associated with a predisposition to pneumococcal carriage, we measured the concentrations of 30 cytokines and of proteins, including interleukin 1 receptor antagonist (IL-1RA), in nasal lining fluid (Fig. 1b). After multiple-testing correction, we found that at day 0 , directly prior to S. pneumoniae inoculation, LAIV significantly increased concentrations of 20 cytokines, including CXCL10 (IP-10), tumor-necrosis factor (TNF), interleukin 10 (IL-10), IFN- $\gamma$ and IL-15 (Fig. $1 \mathrm{~b}$ and Supplementary Table 1). In contrast, the control group did not show any significant increase in cytokine response at day 0 . Following $S$. pneumoniae inoculation, $S$. pneumoniae carriage in the absence of LAIV was associated with increased concentration of epidermal growth factor at day 2 and decreased concentration of IL-1RA at day 9 after $S$. pneumoniae inoculation (compared with that at baseline), neither of which remained significant after multiple-testing correction. No other cytokines, including IL-17A or CCL2, were significantly altered by carriage alone (Fig. 1b).

Even before bacterial inoculation, nasal inflammatory responses to LAIV differed between those who went on to become carriers and those who were protected from carriage (Fig. 1c). In particular, IL-10 was significantly increased in LAIV-vaccinated subjects who did not acquire S. pneumoniae following inoculation (5.8-fold 


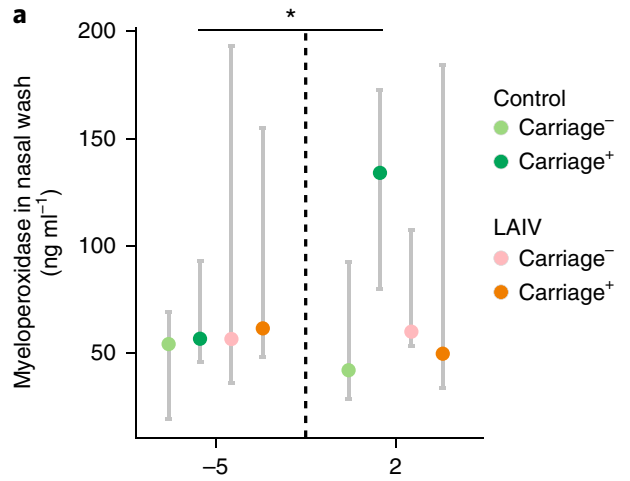

Time after S. pneumoniae inoculation (d)

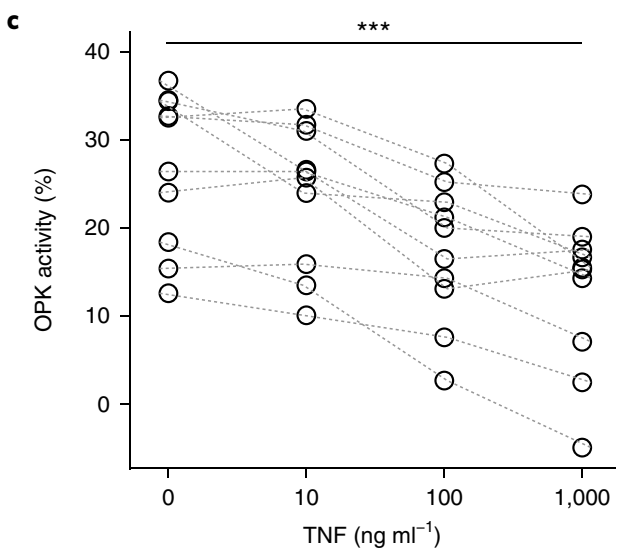

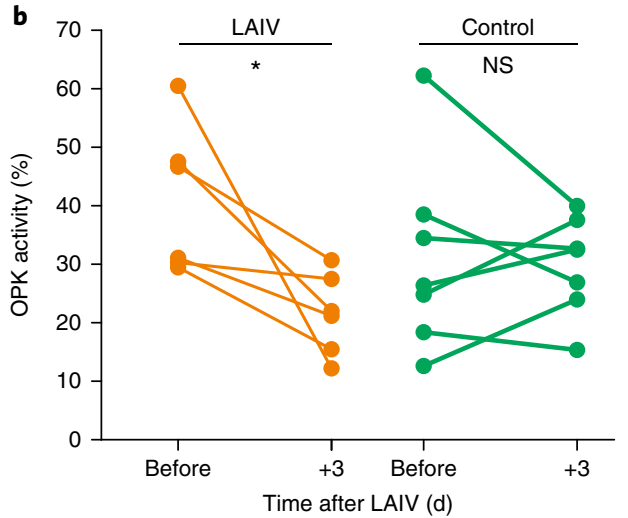

d

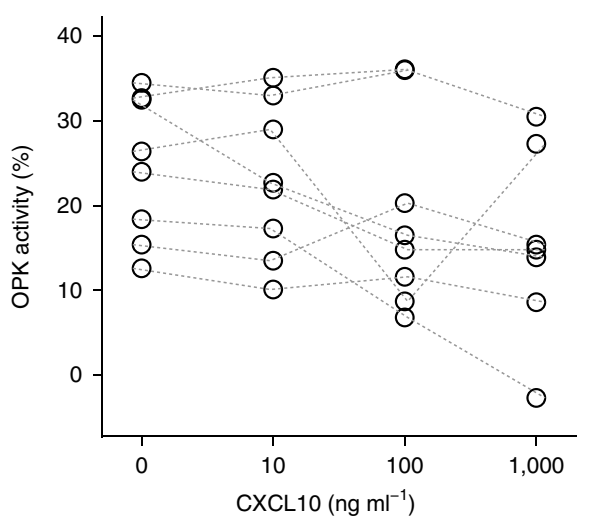

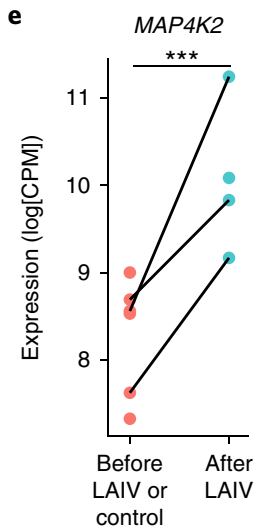
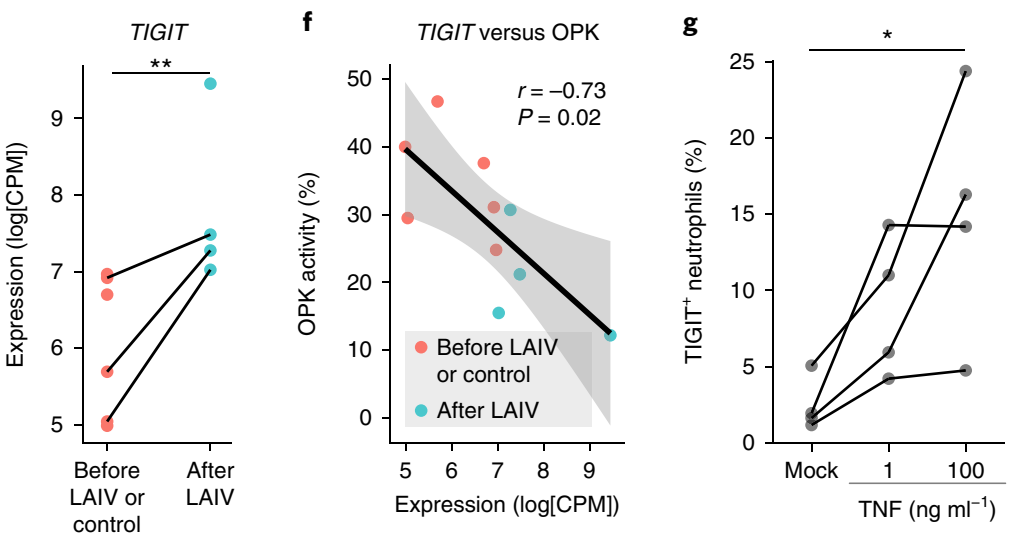

Fig. 2 | Neutrophil function is impaired following LAIV administration. a, Concentrations of myeloperoxidase in nasal wash of volunteers before or 2 days after S. pneumoniae inoculation. Median and interquartile range are shown (for $n=9$ LAIV carriage $^{-}$and LAIV carriage ${ }^{+}$and for $n=10$ control carriage ${ }^{-}$and control carriage ${ }^{+}$subjects). ${ }^{\star} P=0.014$ by two-tailed Wilcoxon paired test. $\mathbf{b}$, S. pneumoniae OPK capacity of blood neutrophils before and 3 days following LAIV $(n=6)$ or control (tetravalent inactivated influenza vaccine or no, $n=7)$ vaccination. Individual subjects are shown and connected by lines. ${ }^{\star} P=0.031$ by two-tailed Wilcoxon paired test. NS, not significant. $\mathbf{c}, \mathbf{d}$, Effect of exogenous TNF (c; $n=10)$ and CXCL10 (d; $n=8)$ on OPK activity of blood neutrophils of healthy volunteers. ${ }^{\star \star} P=1.15 \times 10^{-5}$ by Friedman test. Neutrophils from six subjects were used in three independent experiments. Individual samples are depicted and connected by dashed lines. e, Normalized MAP4K2 and TIGIT counts on sorted neutrophils before LAIV or in control arm ( $n=6$, red) and following LAIV ( $n=4$, blue). Individual samples are shown, and paired samples are connected by black lines. ${ }^{\star \star} P=0.008$ and ${ }^{\star \star \star} P=3.2 \times 10^{-5}$ twotailed unadjusted $P$ values using a negative binomial generalized linear model (DESeq2). f, Correlation between OPK activity and TIGIT counts $(n=10)$. Spearman rho $(r)$ and $P$ value are shown. Regression line and 95\% confidence intervals (shaded area) are shown. $\mathbf{g}$, Levels of TIGIT on blood neutrophil surface measured by flow cytometry after a 30-min incubation without or with $1 \mathrm{ng} \mathrm{ml^{-1 }} \mathrm{TNF}$ or $100 \mathrm{ng} \mathrm{ml}^{-1} \mathrm{TNF}(n=4)$. ${ }^{\star} P=0.042$ by Friedman test. Individual subjects are depicted by dots and connected by lines.

increase), but not in those who became carriers following inoculation (2.0-fold increase). In contrast, CXCL10 was significantly increased in subjects who went on to become carriers (2.4-fold increase), but not in those who remained carriage-negative (1.5fold increase). Moreover, subjects with increased concentrations of
CXCL10 before inoculation displayed higher pneumococcal load following S. pneumoniae inoculation (Fig. 1d). This suggests that differences in the response to influenza virus are associated with secondary susceptibility to $S$. pneumoniae. To test whether this was specific for LAIV infection, we measured CXCL10 in nasal washes 
from an independent cohort in which a subset of subjects had an asymptomatic viral upper respiratory tract infection the week before $S$. pneumoniae inoculation that did not progress to symptomatic infection. These comprised infections with rhinovirus $(n=12)$, coronavirus $(n=5)$, respiratory syncytial virus $(n=2)$ and parainfluenzavirus $(n=1)^{29}$. The predominant virus, rhinovirus, was recently shown to associate with increased pneumococcal acquisition and transmission ${ }^{30}$. In these virus-infected subjects, CXCL10 concentration was increased (Supplementary Fig. 2), and baseline CXCL10 concentration correlated with increased pneumococcal load also in this second cohort (Fig. 1e). Unfortunately, sample sizes were too small to further investigate in depth the effects of infection with the different viruses, which are likely to have divergent consequences. Although the correlation was modest in this validation cohort, suggesting that other host and environmental factors are involved, we identified a biomarker, CXCL10, that was predictive of S. pneumoniae load.

Early neutrophil degranulation in response to carriage is impaired by LAIV infection. In mouse models, neutrophil recruitment after onset of carriage contributes to control of the bacteria ${ }^{15}$. We observed pre-existing high numbers of neutrophils in the human nasal lining, and pneumococcal carriage did not lead to significant further recruitment of neutrophils (Supplementary Fig. 3a,b). To investigate whether luminal neutrophils were involved in the early control of carriage, we measured concentrations of myeloperoxidase, a marker for neutrophil degranulation ${ }^{31}$, in nasal washes. Concentrations were increased (2.2-fold) at 2 days after challenge in control carriage ${ }^{+}$but not carriage ${ }^{-}$individuals (Fig. 2a). This neutrophil activation was impaired in the LAIV group, who displayed high carriage load during early carriage and had increased acquisition compared with that of controls. Together, these findings suggest that neutrophil degranulation is important for the initial control of carriage. To investigate whether neutrophils were also impaired systemically following LAIV, as reported during wild-type influenza infection $^{32}$, we isolated blood neutrophils before, and at 3 days after, LAIV administration from a subset of subjects. We confirmed that opsonophagocytic killing (OPK) of S. pneumoniae by blood neutrophils was decreased following LAIV (Fig. 2b). This effect could be mimicked by the addition of TNF, but not CXCL10, to neutrophils from healthy donors in vitro, decreasing killing capacity in a dosedependent manner (Fig. 2c,d). NanoString expression analysis of 594 genes revealed ten differentially expressed genes in blood neutrophils 3 days post-LAIV (Supplementary Table 2). Among those were the genes encoding MAP4K2 (mitogen-activated protein kinase kinase kinase kinase 2; 3.2-fold increase), which acts on the TNF signal-transduction pathway ${ }^{33}$, and the co-inhibitory receptor TIGIT ( $\mathrm{T}$ cell immunoreceptor with immunoglobulin and ITIM domains; 3.6-fold increase; Fig. 2e). TIGIT expression levels were also negatively correlated with neutrophil killing capacity $(r=-0.73$; Fig. 2f). TIGIT is an immunological checkpoint protein that has been described to promote regulatory $\mathrm{T}$ cell function ${ }^{34}$, but its expression on neutrophils has not been previously appreciated, to our knowledge. Incubation of whole blood with recombinant TNF increased TIGIT levels on neutrophil surfaces within $30 \mathrm{~min}$ in a dose-dependent manner (Fig. 2g).

Taken together, these findings indicate that inflammation following LAIV impairs the response of nasal and systemic neutrophils to pneumococcus, which could be mimicked by addition of exogenous TNF to neutrophils and is associated with an upregulation of TIGIT.

Pneumococcal carriage-induced monocyte recruitment to the nose is impaired by LAIV infection. Immunophenotyping revealed a significant recruitment of monocytes to the nose following establishment of carriage (Fig. 3a and Supplementary Fig. 4). Monocyte numbers increased as early as 2 days following $S$. pneumoniae inoculation,

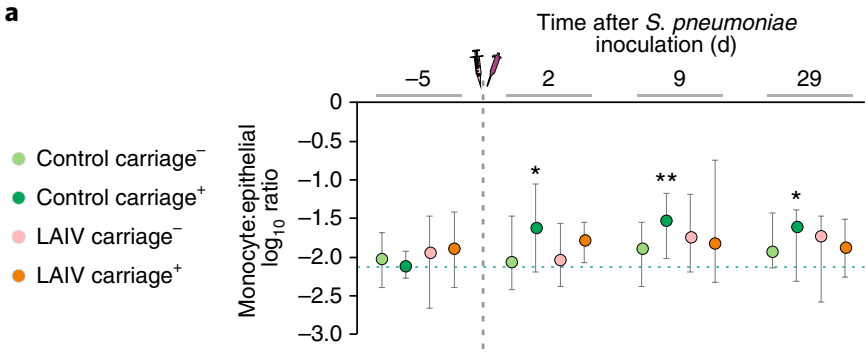

b

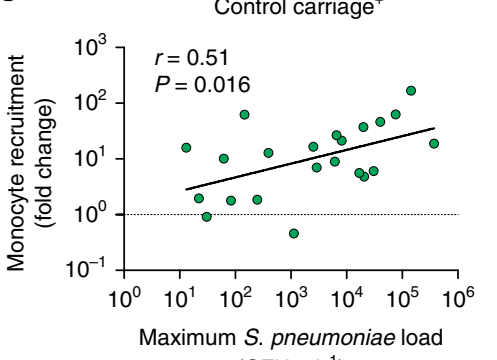

$\left(\mathrm{CFU} \mathrm{ml^{-1 }}\right.$ )
C

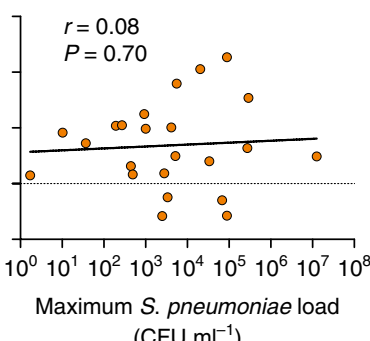

$\left(\mathrm{CFU} \mathrm{m}{ }^{-1}\right.$ )
Fig. 3 | Monocyte recruitment following pneumococcal colonization is impaired during LAIV co-infection. a, Median and interquartile range of nasal monocyte numbers normalized to epithelial cell numbers are shown for control carriage ${ }^{+}(n=24)$, control carriage ${ }^{-}(n=37)$, LAIV carriage $^{+}(n=25)$ and LAIV carriage ${ }^{-}(n=30)$ groups. The dashed green line shows the baseline level in the control carriage ${ }^{+}$group. ${ }^{\star} P=0.038$ at day 2 and $P=0.030$ at day $29 ;{ }^{\star \star} P=0.002$ by two-tailed Wilcoxon paired non-parametric test. $\mathbf{b}, \mathbf{c}$, Levels of maximum pneumococcal $(S$. pneumoniae) load in nasal wash are shown for the control group $(\mathbf{b} ; n=22)$ and LAIV group $(\mathbf{c} ; n=23)$ and are correlated with the maximum monocyte recruitment (fold change to baseline). Individual subjects are shown, and Spearman correlation analysis is shown.

peaked at 9 days (median $4.8 \times$ increase) and remained elevated 29 days after $S$. pneumoniae inoculation. In contrast, there was no recruitment of $\mathrm{CD}^{+} \mathrm{T}$ cells to the nose (Supplementary Fig. $4 \mathrm{~b}$ ). LAIV infection prior to pneumococcal carriage impaired the recruitment of monocytes to the nose (Fig. 3a). Moreover, peak pneumococcal load was associated with increased monocyte recruitment in the control group, but not in the LAIV group (Fig. 3b,c). Indeed, for subjects in the control group with very low carriage densities, which were detectable only by molecular methods, no monocyte recruitment was observed (Supplementary Fig. 4c). This suggests that a minimum S. pneumoniae load is required for sensing and monocyte recruitment and that LAIV infection interferes with this process. Although CCL2 (MCP-1) was not substantially induced following $S$. pneumoniae carriage, its concentration was correlated with numbers of monocytes at all time points (Supplementary Fig. 5a). Furthermore, stratification of individuals showed that those with increased CCL2 concentration at day 2 after S. pneumoniae inoculation exhibited increased monocyte recruitment (Supplementary Fig. 5b). Concentrations of IL-6, IFN- $\gamma$ and TNF were also correlated with numbers of monocytes at each time point, but stratification of individuals did not reveal differential recruitment of monocytes (Supplementary Fig. 5a,b). In a second, independent cohort that did not receive any vaccine, monocytes were increased at day 9 following $S$. pneumoniae inoculation, which was correlated with an increased CCL2 concentration in nasal fluid, validating these results (Supplementary Table 3 and Supplementary Fig. 5c).

Thus, acquisition of pneumococcal carriage led to a recruitment of monocytes to the nasopharynx, a process that was associated with pneumococcal load and CCL2 induction and that was inhibited by LAIV infection. 


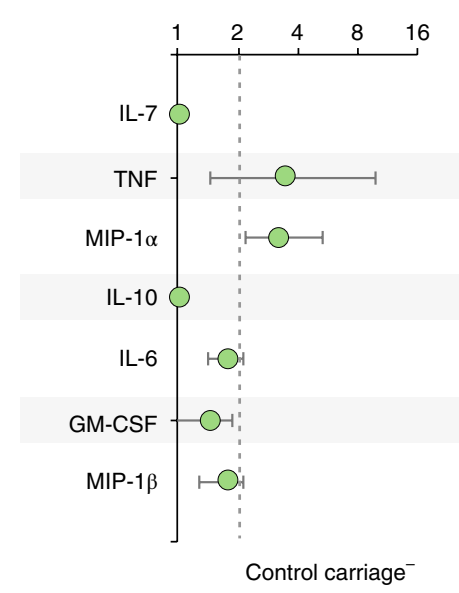

Cytokine production (fold change to unstimulated)

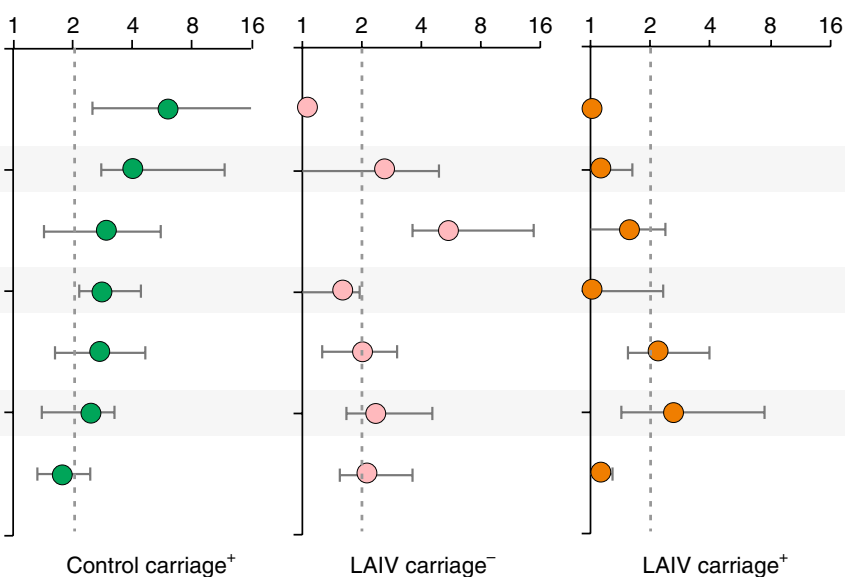

b
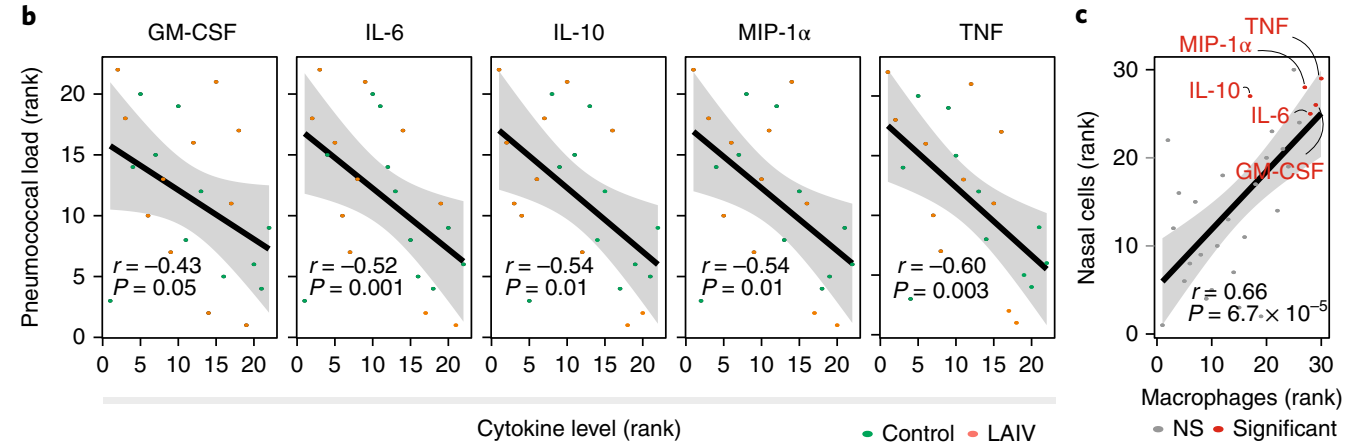

Fig. 4 | Pneumococcus-specific responses are induced following colonization, which is impaired by LAIV co-infection. a, Whole nasal cells were collected from 48 subjects 28 days post-inoculation and stimulated for $18 \mathrm{~h}$ with heat-killed S. pneumoniae. Supernatant was collected, and concentrations of 30 cytokines were measured by multiplex enzyme-linked immunosorbent assay. The median and interquartile range for cytokines induced at least twofold in at least one condition are displayed. b, Correlations between cytokine production following S. pneumoniae stimulation and pneumococcal load are shown $(n=22)$. Spearman non-parametric correlation test results and regression lines with shaded $95 \%$ confidence intervals are shown per cytokine. c, The cytokine profile from alveolar macrophages (median for six volunteers shown) exposed to $\mathrm{S}$. pneumoniae for $18 \mathrm{~h}$ was compared with that of stimulated whole nasal cells (median of control carriage ${ }^{+}$group shown). Spearman non-parametric correlation test results and regression lines with shaded $95 \%$ confidence intervals are shown.

Nasal responses associated with pneumococcal clearance are impaired by LAIV. To assess anti-pneumococcal responses induced by carriage, we collected nasal cells 29 days after S. pneumoniae inoculation, stimulated them in vitro with heat-killed $S$. pneumoniae and measured concentrations of 30 cytokines in the supernatant. Increased production (fold change $>2$ and $q<0.05$ ( $P$ value corrected for multiple testing) to unstimulated control) of TNF, macrophage inflammatory protein $1 \alpha$ (MIP-1 $\alpha)$, IL-10, IL-6 and granulocyte-macrophage colony-stimulating factor upon restimulation was observed in the control carriage ${ }^{+}$group (Fig. $4 \mathrm{a}$ and Supplementary Fig. 6a). In the LAIV carriage $^{+}$group, however, this boosting of anti-pneumococcal cytokine responses by rechallenge was absent (Fig. 4a and Supplementary Fig. 6a). The production of the above five cytokines correlated with decreased pneumococcal load at day 29 after $S$. pneumoniae inoculation, suggesting these responses are involved in $S$. pneumoniae clearance (Fig. 4b). To test whether monocytes/macrophages were the source of these cytokines, we compared the cytokine signature from whole nasal cells with that from alveolar macrophages exposed to $S$. pneumoniae in vitro (Fig. 4c). Relative cytokine production was highly correlated between the two cell populations, suggesting that nasal monocytes/ macrophages could be the source of these cytokines. This is supported by the observation that in carriers with low carriage load (detectable only by molecular methods), absence of monocyte recruitment was associated with absent $S$. pneumoniae-specific responses (Supplementary Fig. 6b).

In conclusion, carriage led to increased responses of nasal cells to pneumococcal stimulation, which was potentially due to the infiltration of monocytes. This was impaired by prior LAIV infection and was correlated with clearance of pneumococcal carriage (Supplementary Fig. 7).

LAIV alters nasal gene-expression responses to carriage. To identify gene signatures associated with the observed responses to pneumococcal carriage and infection with LAIV, we performed RNA sequencing on whole nasal cells at days $-5,2$ and 9 after $S$. pneumoniae inoculation (Fig. 5 and Supplementary Table 4). Carriage without LAIV induced 834 and 176 differentially expressed genes (DEGs) at days 2 and 9, respectively (Fig. 5a). These genes were enriched for pathways associated with gap junction trafficking and regulation (including GJA1, TJP1 and multiple GJB genes) and degradation of the extracellular matrix (including COL17A1, COL12A1, $L A M A 3$ and $K L K 7)$. In the carriage ${ }^{-}$group, a smaller number of DEGs was observed (161 and 248 at days 2 and 9 , respectively).

In the LAIV carriage ${ }^{+}$group, 936 and 711 DEGs were observed at day 2 and day 9, respectively. Surprisingly, despite the high concentrations of inflammatory cytokines observed in the LAIV carriage $^{-}$group, only a relatively small number of DEGs were observed 


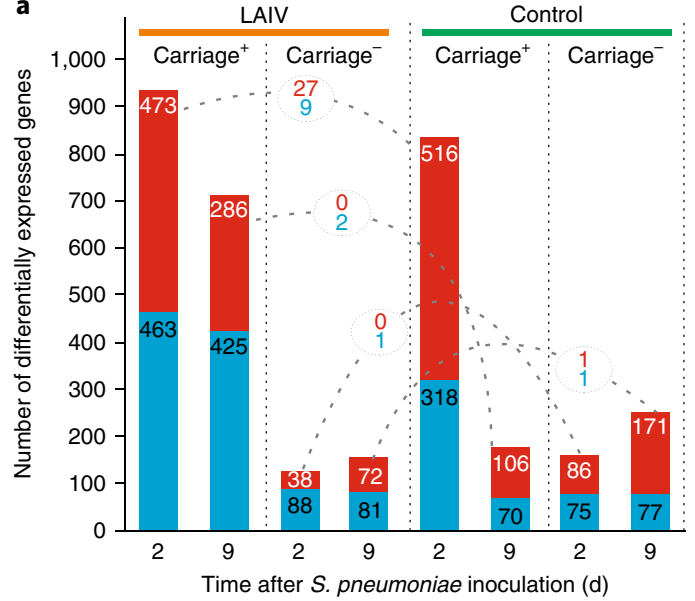

b

Downregulated

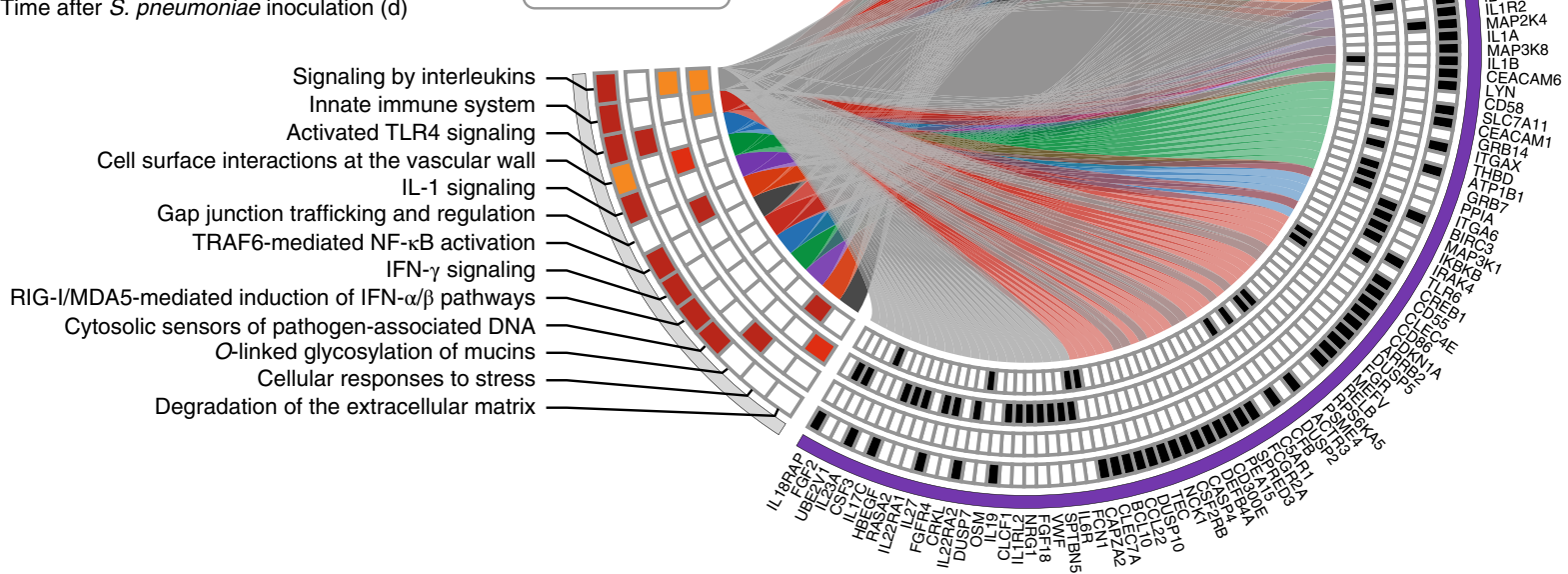

Fig. 5 | Nasal transcriptomics following LAIV-S. pneumoniae co-infection $(\boldsymbol{n}=\mathbf{3 5}$ ). $\mathbf{a}$, The number of differentially expressed genes (DEGs) between each time point and the baseline for each group are shown. Upregulated and downregulated genes are depicted in red and blue, respectively. Connections between bars show the number of common genes between LAIV and control conditions, where colors reflect distinct pathways. b, Circular representation of DEGs and gene set enrichment analysis for LAIV carriage ${ }^{+}$and control carriage+ groups at days 2 and 9 after S. pneumoniae inoculation. The individual $\log _{2}$ [fold change] values (baseline-normalized) were used as ranks in a single sample gene set enrichment analysis to identify consistently enriched pathways among subjects. Genes and pathways are connected by lines.

at days 2 and 9 (126 and 153, respectively). DEGs of carriage ${ }^{+}$subjects receiving LAIV and DEGs of carriage ${ }^{+}$subjects without LAIV showed very little overlap, with only 38 DEGs at day 2 and 2 DEGs at day 9 in common. Very little overlap was observed at the pathway level between these groups, indicating LAIV alters the natural responses to pneumococcus (Fig. 5b and Supplementary Table 5). This could reflect transcriptome kinetics, due to altered differentiation and cellular activation, or it could reflect changes in cell migration to the nasal mucosa.

The LAIV carriage ${ }^{+}$group showed enrichment for genes encoding molecules involved in the Toll-like receptor 3 (TLR3) signaling cascade, in retinoic acid-inducible gene I (RIG-I)/melanoma differentiation-associated protein 5 (MDA5)-mediated induction of IFN- $\alpha / \beta$ pathways and in IFN- $\gamma$ signaling, which is in agreement with the induction of antiviral responses following LAIV vaccination $^{35}$. Moreover, TLR4 signaling was also enriched in this group. The pneumococcal protein pneumolysin is sensed by TLR $4^{36}$, and it is possible that the increased pneumococcal load following LAIV vaccination led to increased pneumolysin sensing. $O$-linked glycosylation of mucins, which are used by $S$. pneumoniae as a carbohydrate source for growth ${ }^{37}$, was also enriched in the LAIV carriage $^{+}$group (including the genes ST3GAL4, GALNT7, GCNT3 and B4GALT5). ST3GAL4 encodes a sialyl transferase, and cleavage of sialic acids by the influenza neuraminidase has previously been shown to promote pneumococcal growth ${ }^{38}$. This finding supports a
LAIV-mediated effect on pneumococcal growth through alterations of host factors. Common genes and pathways between the LAIVvaccinated carriers and control carriers include innate immune system' and 'signaling by interleukins' (IL1B, CLEC4E, CD55 and $I L 1 R N)$. In conclusion, the genome-wide transcriptomic response to pneumococcal carriage was substantially altered on both the gene level and the pathway level by LAIV.

Gene modules associated with recruitment of monocytes. To identify sets of coexpressed genes post-LAIV and carriage, we used CEMiTool on the baseline-normalized data of LAIV and control groups, separately ${ }^{39}$. This modular expression analysis revealed genes that may act together or are similarly regulated during the immune responses to carriage and infection.

Genes in the control cohort were grouped into four coexpression modules, of which three were significantly enriched for known reactome pathways (Supplementary Data 1). Module M1 was enriched in the carriage ${ }^{+}$group at day 9 after $S$. pneumoniae inoculation (Fig. 6a). Numbers of monocytes were correlated with the average fold change count in this module, suggesting that these genes reflect the infiltration of monocytes (Fig. 6b). To further investigate these monocytes, we performed gene set enrichment analysis on the module M1 genes using a list of genes from distinct monocyte subsets (Fig. $6 \mathrm{c})^{40}$. These genes were enriched for classical CD14 ${ }^{+} \mathrm{CD} 16$ monocytes and not for other monocyte subsets. Moreover, this 
a

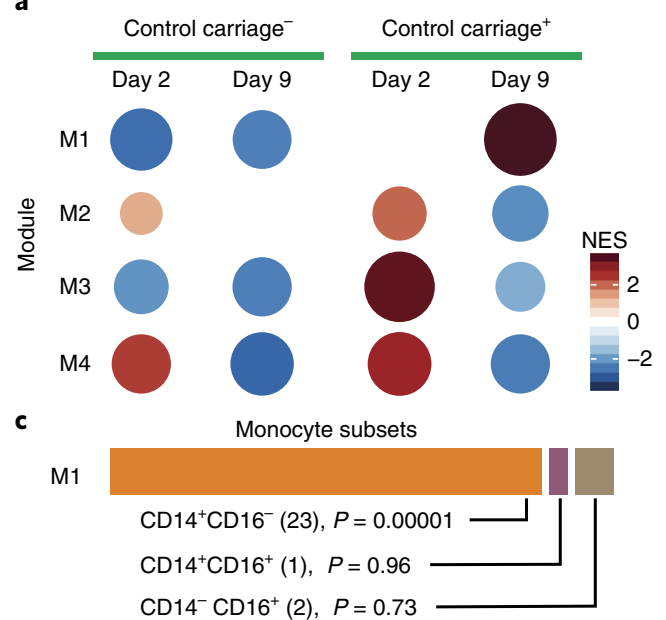

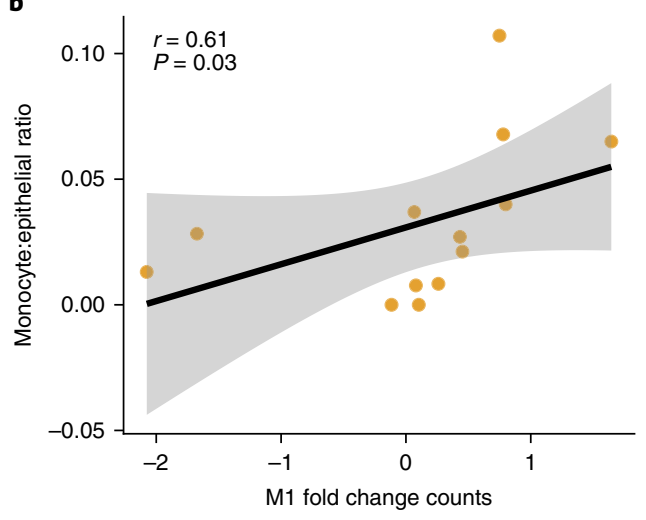

e

e $\quad \mathrm{M} 1$

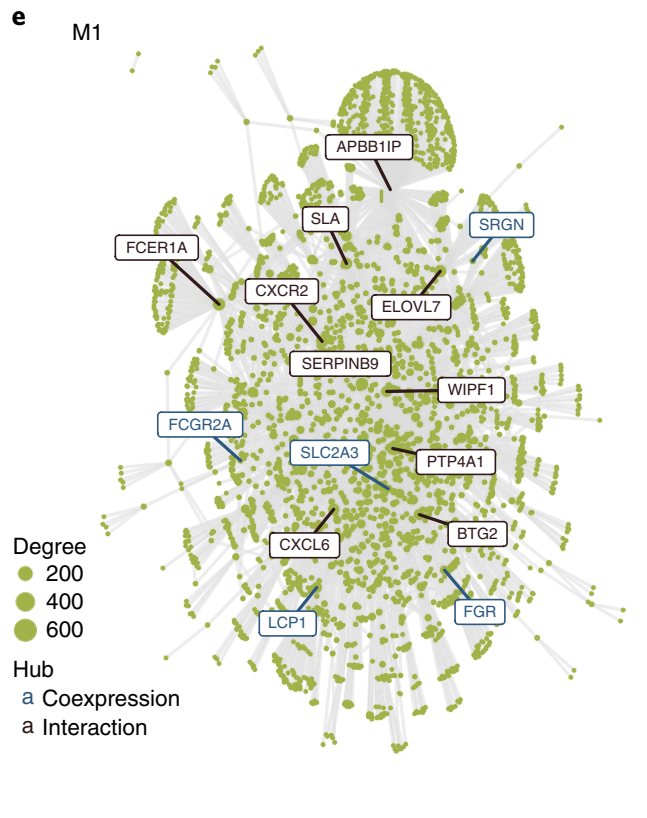

Fig. 6 | CEMiTool applied to control cohort: module M1. Raw counts were normalized using log[CPM], and $\log _{2}$ [fold change] values were calculated for each time point against the baseline after which coexpression modules were extracted. $\mathbf{a}$, Gene set enrichment analyses showing the module activity at each time point for carriage ${ }^{+}$and carriage ${ }^{-}$groups. Symbol size and color reflect normalized enrichment score (NES). b, Correlation with average fold change counts of all M1 genes at day 9 with paired numbers of monocytes from the volunteer's other nostril. Individual subjects are shown, along with regression line with 95\% confidence interval and Spearman correlation analysis $(n=13)$. c, The genes of M1 present with genes highly expressed in $\mathrm{CD}_{14}{ }^{+} \mathrm{CD} 16^{-}$( 578 genes), $\mathrm{CD} 14^{+} \mathrm{CD}_{16}{ }^{+}$(108 genes) and CD14-CD16+ (162 genes) ${ }^{40}$, showing the overlapping number of genes between $\mathrm{M} 1$ and monocyte subsets in parentheses. The overlap for significance was analyzed using the $\chi^{2}$ test. $\mathbf{d}$, Over-representation analysis of module M1 using gene sets from the Reactome Pathway database. e, Interaction plot for M1, with gene nodes highlighted.

module was enriched for genes related to 'chemokine receptors bind chemokines' and 'IFN- $\alpha / \beta$ signaling' (Fig. $6 \mathrm{~d}$ ). Type I interferons have been shown to be required for the clearance of pneumococcal carriage in mouse models ${ }^{41}$, and these findings suggest that their activity in monocytes might be critical for this. CEMiTool also integrates coexpression analysis with protein-protein interaction data. Expression of the gene encoding the chemokine CXCL6 and that of the gene encoding its receptor CXCR2 were identified as hubs in module M1 (Fig. 6e and Supplementary Data 1). CXCR2 engagement has been shown to induce attachment of monocytes to the endothelial layer, initiating chemotaxis, which suggests this interaction could contribute to monocyte recruitment ${ }^{42}$. Module M3 was enriched for genes related to 'extracellular matrix organization' and 'collagen formation' (Fig. 7).

For LAIV, we identified six distinct coexpression modules (Supplementary Data 2), which were strongly enriched for genes related to 'diseases associated with $O$-glycosylation of proteins' (module M1), 'immunoregulatory interactions between a lymphoid and a non-lymphoid cell' (module M3), 'chemokine receptors bind chemokines' (module M4) and 'interferon signaling' (module M5; Fig. 8). Indeed, the hubs of module M5 are well known type I interferon-related genes, such as ISG15, OAS1, OASL, IFIT1-3 and IFITM1. Taken together, our findings reveal that a strong local antiviral response is elicited in response to LAIV infection.

\section{Discussion}

This study addresses fundamental questions about the immune responses that control and clear S. pneumoniae carriage and how influenza infection can alter this control. By using a double experimental human challenge model with LAIV and S. pneumoniae, we revealed that $S$. pneumoniae carriage led to quick degranulation of pre-existing nasal neutrophils in the human nose and recruitment 
a

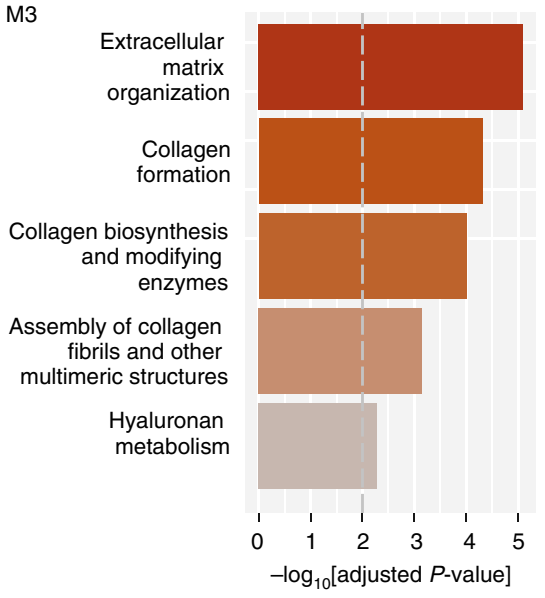

b

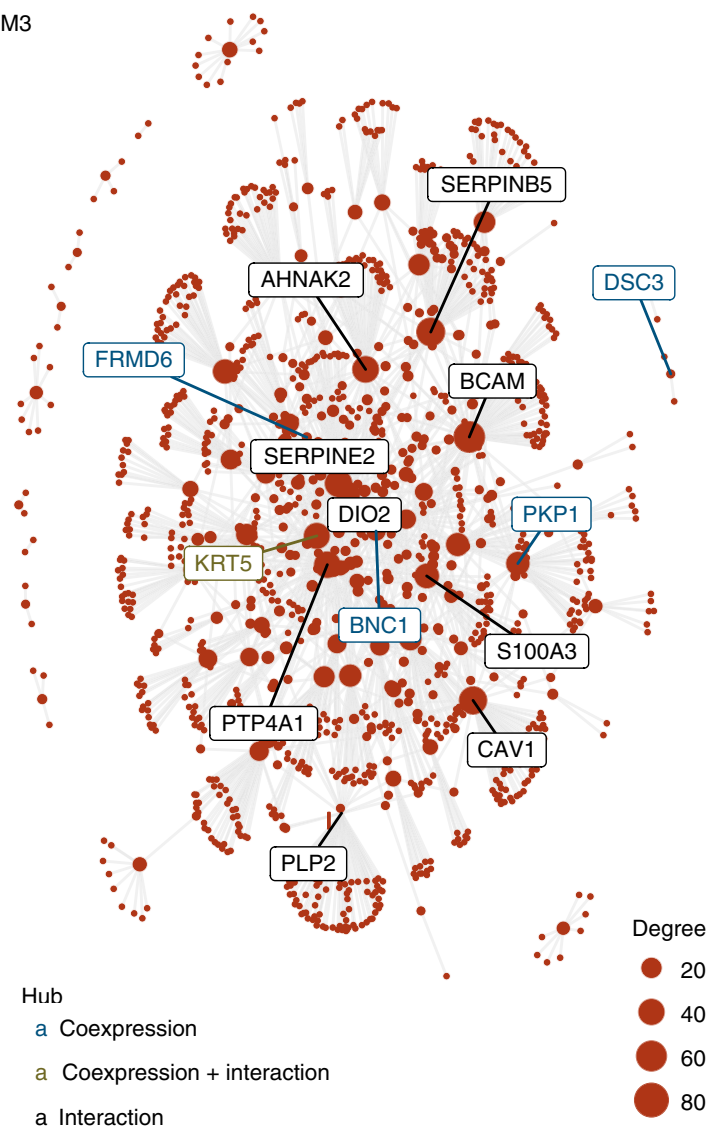

Fig. 7 | CEMiTool applied to control group: module M3. Raw counts were normalized using log[CPM], and $\log _{2}$ [fold change] values were calculated for each time point against the baseline after which coexpression modules were extracted. a, Over-representation analysis of module M3 of the control group using gene sets from the Reactome Pathway database. b, Interaction plot for M3, with gene nodes highlighted.

of monocytes, promoting bacterial clearance. LAIV infection impaired these immune responses following carriage. LAIV is an attenuated influenza strain, and wild-type influenza viruses might have even more pronounced effects on the host response to pneumococcus. Carriage in the absence of LAIV was associated with only limited inflammation, corroborating the view of $S$. pneumoniae as a commensal bacterium that can asymptomatically colonize healthy adults ${ }^{43}$. In contrast, robust proinflammatory cytokine responses were measured following LAIV at both the protein- and gene-expression level. These results provide an explanation for the published finding that LAIV increased acquisition of $S$. pneumoniae and carriage load ${ }^{14}$.

In addition, our findings that LAIV led to impaired blood neutrophil killing capacity and that the addition of TNF, the nasal levels of which were increased following LAIV, to neutrophils in vitro impaired their activity highlight the crucial role of neutrophils in susceptibility to secondary bacterial infection ${ }^{44}$. The association of TIGIT with this impaired neutrophil function following influenza infection warrants further investigation, as TIGIT-blocking therapeutics are currently being developed for treatment of cancer and human immunodeficiency virus ${ }^{45}$.

We identified CXCL10 as a marker for increased susceptibility to $S$. pneumoniae, and we propose that this should be further investigated as a potential therapeutic target for secondary bacterial infections associated with viral infections. Our data showed that individuals with higher concentrations of CXCL10 prior to $S$. pneumoniae inoculation had higher bacterial loads. In a previous study, children with pneumonia with viral and bacterial (predominantly pneumococcal) co-infection had increased amounts of CXCL10 compared with those of children with only viral or bacterial pneumonia, a finding that is associated with disease severity ${ }^{46}$. Data in mice suggest that CXCL10 plays a direct role during pneumonia. Mice with genetic ablation of CXCR3, the receptor for CXCL10, CXCL9 and CXCL11, showed increased survival, decreased lung inflammation and less invasion following infection, depending on the pneumococcal inoculation strain used ${ }^{47}$. Moreover, addition of exogenous CXCL10 prior to infection of mice with influenza virus or respiratory syncytial virus increased pneumonia severity ${ }^{48}$.

Our results support previous findings from mouse models showing that CCL2 signaling and monocyte recruitment are key mediators of pneumococcal carriage clearance ${ }^{16}$. However, contrary to key mechanisms described in mouse models, we did not observe any production of IL-17A or neutrophil recruitment to the nose following carriage or associated with carriage clearance ${ }^{15-17}$, underlining the importance of confirming mouse findings with human data.

One limitation of this study is that only one pneumococcal serotype $6 \mathrm{~B}$ isolate was used; future studies using other isolates with a more or less invasive phenotype will be able to address how generalizable these findings are across pneumococcal isolates. Nonetheless, the observation that carriage load and duration decline in parallel for all serotypes following repeated exposure suggests that immunological control of newly acquired S. pneumoniae is mediated by similar mechanisms independent of the colonizing serotype $\mathrm{e}^{49}$. 


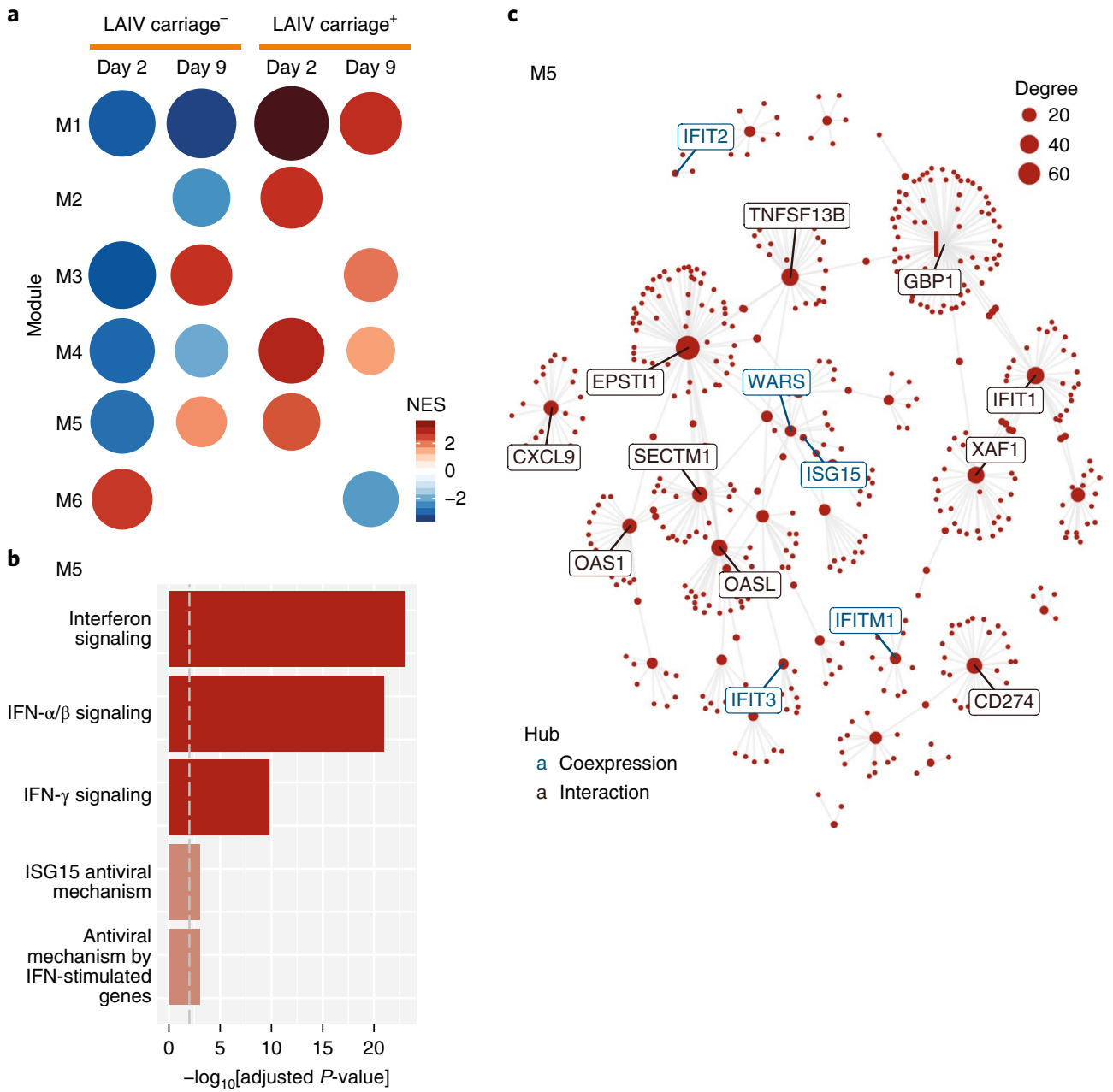

Fig. 8 | CEMiTool applied to LAIV. Raw counts were normalized using log[CPM], and $\log _{2}[$ fold change] values were calculated for each time point against the baseline after which coexpression modules were extracted. $\mathbf{a}$, Gene set enrichment analyses showing the module activity at each time point for carriage+ and carriage- LAIV groups. b, Over-representation analysis of module M5 of the LAIV group using gene sets from the Reactome Pathway database. c, Interaction plot for M5, with gene nodes highlighted.

In conclusion, this study highlights the importance of innate immunity-which was impaired by pre-existing viral infectionsin the control of carriage load and clearance of S. pneumoniae. Secondary bacterial infection following viral respiratory tract infection creates a large disease burden worldwide, and disrupting viralbacterial synergy through host-directed therapy could prove an attractive addition to current therapeutic and vaccination options ${ }^{50}$.

\section{Online content}

Any methods, additional references, Nature Research reporting summaries, source data, statements of data availability and associated accession codes are available at https://doi.org/10.1038/ s41590-018-0231-y

Received: 20 June 2018; Accepted: 7 September 2018;

Published online: 29 October 2018

\section{References}

1. Liu, L. et al. Global, regional, and national causes of under-5 mortality in 2000-15: an updated systematic analysis with implications for the Sustainable Development Goals. Lancet 388, 3027-3035 (2016).

2. Morens, D. M., Taubenberger, J. K. \& Fauci, A. S. Predominant role of bacterial pneumonia as a cause of death in pandemic influenza: implications for pandemic influenza preparedness. J. Infect. Dis. 198, 962-970 (2008).
3. Goldblatt, D. et al. Antibody responses to nasopharyngeal carriage of Streptococcus pneumoniae in adults: a longitudinal household study. J. Infect. Dis. 192, 387-393 (2005).

4. Simell, B. et al. The fundamental link between pneumococcal carriage and disease. Expert Rev. Vaccines 11, 841-855 (2012).

5. Melegaro, A., Gay, N. J. \& Medley, G. F. Estimating the transmission parameters of pneumococcal carriage in households. Epidemiol. Infect. 132, 433-441 (2004).

6. Ferreira, D. M. et al. Controlled human infection and rechallenge with Streptococcus pneumoniae reveals the protective efficacy of carriage in healthy adults. Am. J. Respir. Crit. Care Med. 187, 855-864 (2013).

7. McCool, T. L., Cate, T. R., Moy, G. \& Weiser, J. N. The immune response to pneumococcal proteins during experimental human carriage. J. Exp. Med. 195, 359-365 (2002).

8. Mina, M. J. \& Klugman, K. P. The role of influenza in the severity and transmission of respiratory bacterial disease. Lancet Respir. Med. 2, 750-763 (2014).

9. Alpkvist, H. et al. Clinical and microbiological factors associated with high nasopharyngeal pneumococcal density in patients with pneumococcal pneumonia. PLoS ONE 10, e0140112 (2015).

10. Wolter, N. et al. High nasopharyngeal pneumococcal density, increased by viral coinfection, is associated with invasive pneumococcal pneumonia. J. Infect. Dis. 210, 1649-1657 (2014).

11. Albrich, W. C. et al. Pneumococcal colonisation density: a new marker for disease severity in HIV-infected adults with pneumonia. BMJ Open 4, e005953 (2014).

12. Thors, V. et al. The effects of live attenuated influenza vaccine on nasopharyngeal bacteria in healthy 2 to 4 year olds. A randomized controlled trial. Am. J. Respir. Crit. Care Med. 193, 1401-1409 (2016). 
13. Mina, M. J., McCullers, J. A. \& Klugman, K. P. Live attenuated influenza vaccine enhances colonization of Streptococcus pneumoniae and Staphylococcus aureus in mice. mBio 5, e01040-13 (2014).

14. Rylance, J. et al. Effect of live attenuated influenza vaccine on pneumococcal carriage. bioRxiv https://doi.org/10.1101/343319 (2018).

15. $\mathrm{Lu}, \mathrm{Y}$. J. et al. Interleukin-17A mediates acquired immunity to pneumococcal colonization. PLoS Pathog. 4, e1000159 (2008).

16. Zhang, Z., Clarke, T. B. \& Weiser, J. N. Cellular effectors mediating Th17-dependent clearance of pneumococcal colonization in mice. J. Clin. Investig. 119, 1899-1909 (2009).

17. Lu, Y. J. et al. GMP-grade pneumococcal whole-cell vaccine injected subcutaneously protects mice from nasopharyngeal colonization and fatal aspiration-sepsis. Vaccine 28, 7468-7475 (2010).

18. Nakamura, S., Davis, K. M. \& Weiser, J. N. Synergistic stimulation of type I interferons during influenza virus coinfection promotes Streptococcus pneumoniae colonization in mice. J. Clin. Investig. 121, 3657-3665 (2011).

19. Sun, K. \& Metzger, D. W. Inhibition of pulmonary antibacterial defense by interferon- $\gamma$ during recovery from influenza infection. Nat. Med 14, 558-564 (2008).

20. Li, W., Moltedo, B. \& Moran, T. M. Type I interferon induction during influenza virus infection increases susceptibility to secondary Streptococcus pneumoniae infection by negative regulation of $\gamma \delta$ T cells. J. Virol. 86, 12304-12312 (2012)

21. Jochems, S. P., Weiser, J. N., Malley, R. \& Ferreira, D. M. The immunological mechanisms that control pneumococcal carriage. PLoS Pathog. 13, e1006665 (2017)

22. Querec, T. D. et al. Systems biology approach predicts immunogenicity of the yellow fever vaccine in humans. Nat. Immunol. 10, 116-125 (2009).

23. Oh, J. Z. et al. TLR5-mediated sensing of gut microbiota is necessary for antibody responses to seasonal influenza vaccination. Immunity 41, 478-492 (2014).

24. $\mathrm{Li}, \mathrm{S}$. et al. Metabolic phenotypes of response to vaccination in humans. Cell 169, 862-877 (2017).

25. Kazmin, D. et al. Systems analysis of protective immune responses to RTS,S malaria vaccination in humans. Proc. Natl Acad. Sci. USA 114 2425-2430 (2017)

26. Nakaya, H. I. et al. Systems biology of vaccination for seasonal influenza in humans. Nat. Immunol. 12, 786-795 (2011).

27. Nakaya, H. I. et al. Systems analysis of immunity to influenza vaccination across multiple years and in diverse populations reveals shared molecular signatures. Immunity 43, 1186-1198 (2015)

28. Li, S. et al. Molecular signatures of antibody responses derived from a systems biology study of five human vaccines. Nat. Immunol. 15, 195-204 (2014).

29. Glennie, S. et al. Modulation of nasopharyngeal innate defenses by viral coinfection predisposes individuals to experimental pneumococcal carriage. Mucosal Immunol 9, 56-67 (2015).

30. Karppinen, S. et al. Acquisition and transmission of Streptococcus pneumoniae are facilitated during rhinovirus infection in families with children. Am. J. Respir. Crit. Care Med. 196, 1172-1180 (2017).

31. Segal, A. W. How neutrophils kill microbes. Annu. Rev. Immunol. 23, 197-223 (2005).

32. Craft, A. W., Reid, M. M. \& Low, W. T. Effect of virus infections on polymorph function in children. Br. Med. J. 1, 1570 (1976).

33. Yuasa, T., Ohno, S., Kehrl, J. H. \& Kyriakis, J. M. Tumor necrosis factor signaling to stress-activated protein kinase (SAPK)/Jun $\mathrm{NH}_{2}$-terminal kinase (JNK) and p38. Germinal center kinase couples TRAF2 to mitogen-activated protein kinase/ERK kinase kinase 1 and SAPK while receptor interacting protein associates with a mitogen-activated protein kinase kinase kinase upstream of MKK6 and p38. J. Biol. Chem. 273, 22681-22692 (1998).

34. Anderson, A. C., Joller, N. \& Kuchroo, V. K. Lag-3, Tim-3, and TIGIT: co-inhibitory receptors with specialized functions in immune regulation. Immunity 44, 989-1004 (2016).

35. Wu, W. et al. RIG-I and TLR3 are both required for maximum interferon induction by influenza virus in human lung alveolar epithelial cells. Virology 482, 181-188 (2015)

36. Malley, R. et al. Recognition of pneumolysin by Toll-like receptor 4 confers resistance to pneumococcal infection. Proc. Natl Acad. Sci. USA 100, 1966-1971 (2003).

37. Paixao, L. et al. Host glycan sugar-specific pathways in Streptococcus pneumonia: galactose as a key sugar in colonisation and infection. PLoS ONE 10, e0121042 (2015). correction 10, e0127483 (2015).
38. Siegel, S. J., Roche, A. M. \& Weiser, J. N. Influenza promotes pneumococcal growth during coinfection by providing host sialylated substrates as a nutrient source. Cell Host Microbe 16, 55-67 (2014).

39. Russo, P. S. T. et al. CEMiTool: a Bioconductor package for performing comprehensive modular co-expression analyses. BMC Bioinformatics 19, 56 (2018)

40. Kwissa, M. et al. Dengue virus infection induces expansion of a CD $14^{+} \mathrm{CD} 16^{+}$ monocyte population that stimulates plasmablast differentiation. Cell Host Microbe 16, 115-127 (2014)

41. Parker, D. et al. Streptococcus pneumoniae DNA initiates type I interferon signaling in the respiratory tract. mBio 2, e00016-00011 (2011).

42. Gerszten, R. E. et al. MCP-1 and IL-8 trigger firm adhesion of monocytes to vascular endothelium under flow conditions. Nature 398, 718-723 (1999).

43. Weiser, J. N. The pneumococcus: why a commensal misbehaves. J. Mol. Med. 88, 97-102 (2010)

44. Rynda-Apple, A., Robinson, K. M. \& Alcorn, J. F. Influenza and bacterial superinfection: illuminating the immunologic mechanisms of disease. Infect. Immun. 83, 3764-3770 (2015).

45. Cox, M. A., Nechanitzky, R. \& Mak, T. W. Check point inhibitors as therapies for infectious diseases. Curr. Opin. Immunol. 48, 61-67 (2017).

46. Hoffmann, J. et al. Viral and bacterial co-infection in severe pneumonia triggers innate immune responses and specifically enhances IP-10: a translational study. Sci. Rep. 6, 38532 (2016).

47. Seyoum, B., Yano, M. \& Pirofski, L. A. The innate immune response to Streptococcus pneumoniae in the lung depends on serotype and host response. Vaccine 29, 8002-8011 (2011)

48. Luo, H., Wang, D., Che, H. L., Zhao, Y. \& Jin, H. Pathological observations of lung inflammation after administration of IP-10 in influenza virus- and respiratory syncytial virus-infected mice. Exp. Ther. Med. 3, 76-79 (2012)

49. Hogberg, L. et al. Age- and serogroup-related differences in observed durations of nasopharyngeal carriage of penicillin-resistant pneumococci. J. Clin. Microbiol. 45, 948-952 (2007).

50. Madhi, S. A., Klugman, K. P. \& Vaccine Trialist Group. A role for Streptococcus pneumoniae in virus-associated pneumonia. Nat. Med. 10, 811-813 (2004)

\section{Acknowledgements}

S.B.G. and D.M.F. are supported by the Medical Research Council (grant MR M011569/1), the Bill and Melinda Gates Foundation (grant OPP1117728) and the National Institute for Health Research Local Comprehensive Research Network. Flow cytometric acquisition was performed on a BD LSR II funded by a Wellcome Trust Multi-User Equipment Grant (104936/Z/14/Z). S.P.J. received support from the Royal Society of Tropical Medicine and Hygiene for NanoString analysis. H.I.N. is supported by the São Paulo Research Foundation (FAPESP; grants 2013/08216-2 and 2012/19278-6). We thank all volunteers for participating in this study, and R. Robinson, C. Lowe, L. Lazarova, K. Piddock and I. Wheeler for clinical support. We also acknowledge M. Mina for his input in study design.

\section{Author contributions}

S.P.J. contributed to conceiving, designing, performing and analyzing experiments and writing the paper. F.M. and H.I.N. contributed to analyzing experiments and writing the paper. B.F.C., M.H., E.M., E.S., J.F.G., C.S., J.Reiné, S.P., E.N., E.L.G., W.A.A.d.S.P. and D.B. contributed to conducting and analyzing experiments. A.H.-W., H.H., C.H., H.A., S.Z., V.C., J.Rylance and S.B.G. contributed to sample collection and/or designing the study. D.M.F. contributed to conceiving, designing and analyzing experiments, designing the study and writing the paper. All authors have read and approved the manuscript.

\section{Competing interests}

The authors declare no competing interests.

\section{Additional information}

Supplementary information is available for this paper at https://doi.org/10.1038/ s41590-018-0231-y.

Reprints and permissions information is available at www.nature.com/reprints. Correspondence and requests for materials should be addressed to S.P.J. or H.I.N. or D.M.F.

Publisher's note: Springer Nature remains neutral with regard to jurisdictional claims in published maps and institutional affiliations.

(c) The Author(s), under exclusive licence to Springer Nature America, Inc. 2018 


\section{Methods}

Study design and sample collection. Healthy adult volunteers were 1:1 randomized to receive either intranasal LAIV (2015/2016 Fluenz Tetra or FluMist Tetra, AstraZeneca) or intramuscular quadrivalent inactivated influenza vaccination (Fluarix Tetra, GlaxoSmithKline), as described previously ${ }^{14}$. The control group also received a nasal saline spray, while the LAIV group also received an intramuscular saline injection. Three days post-vaccination, all subjects were inoculated with 80,000 colony-forming units (CFU) per nostril of $S$. pneumoniae $6 \mathrm{~B}$ type as described previously ${ }^{6,51}$. Nasal microbiopsies (ASL Rhino-Pro, Arlington Scientific) and nasal lining fluid samples (Nasosorption, Hunt Developments) were collected and stored at $-80^{\circ} \mathrm{C}$ as previously described ${ }^{52}$.

Clinical trial details. The double-blinded, randomized clinical LAIV-EHPC (experimental human pneumococcal carriage) trial was registered on EudraCT (number 2014-004634-26) on 28 April 2015 and ISRCTN (number 16993271) on 2 September 2015 and was co-sponsored by the Royal Liverpool University Hospital and the Liverpool School of Tropical Medicine. Key eligibility criteria included capacity to give informed consent, no immunocompromised state or contact with susceptible individuals, no pneumococcal or influenza vaccine or infection in the last 2 years and not having taken part in EHPC studies in the past 3 years. The primary endpoint was the occurrence of pneumococcal colonization as determined by the presence of pneumococcus in nasal wash samples at any time point postinoculation up to and including day 29 , detected using classical microbiology or lytA quantitative PCR as previously described ${ }^{6,51,53}$. In this study, 130 volunteers were inoculated with pneumococcus, giving an $80 \%$ power to identify a $50 \%$ increase in carriage acquisition. Of 130 vaccinated volunteers, 5 were natural pneumococcal carriers ( 2 in the LAIV arm and 3 in the control arm) and were excluded from further analysis. Another 8 subjects in the LAIV arm were excluded following a systematic LAIV dispensing error by a single practitioner, as recommended by the trial steering group. This resulted in final totals of 55 subjects analyzed in the LAIV arm and 62 subjects in the control arm. Key secondary endpoints included the load of pneumococcal colonization in nasal wash samples at each time point following pneumococcal inoculation (days 2, 7, 9, 14, 22 and 29), detected using classical microbiology; the area under the curve of pneumococcal colonization load following pneumococcal inoculation (days 2, 7, 9, 14, 22 and 29), detected using classical microbiology or by molecular methods (lytA); and the immunological mechanisms associated with altered susceptibility to pneumococcus following LAIV. The outcomes reported in this manuscript were a priori included in the study protocol.

Ethics statement. All volunteers gave written informed consent, and research was conducted in compliance with all relevant ethical regulations. Ethical approval was given by the East Liverpool National Health Service Research and Ethics Committee/Liverpool School of Tropical Medicine Research and Ethics Committee, reference numbers 15/NW/0146 and 14/NW/1460 and Human Tissue Authority licensing number 12548 .

Flow cytometry analysis. Immunophenotyping of nasal cells obtained by curettes was performed as previously described ${ }^{52}$. In brief, cells were dislodged from curettes and stained with LIVE/DEAD Fixable Violet Dead Cell Stain (Thermo Fisher Scientific) and an antibody cocktail containing Epcam-PE (9C4; BioLegend), HLADR-PECy7 (L243; BioLegend), CD16-APC (3G8; BioLegend), CD66b-FITC (G10F5, BioLegend), CD3-APCH7 (SK7; BD Biosciences), CD14-PercpCy5.5 (M $\varphi$ P9, BD Biosciences) and CD45-PACOrange (HI30, Thermo Fisher Scientific). Whole blood was stained for $15 \mathrm{~min}$ at room temperature with TIGIT-PECy7 (A15153G, BioLegend) and CD16-APC, followed by $2 \times 10 \mathrm{~min}$ incubation steps with FACSLysis buffer (BD Biosciences) to remove erythrocytes. Samples were acquired on an LSR II flow cytometer (BD Biosciences) and analyzed using Flowjo X (Treestar). Fluorescent minus one controls for each of the included antibodies were used to validate results. For the LAIV and control cohorts, but not the additional validation cohort (Supplementary Fig. 5c), 84 of 553 samples (15.2\%) had less than 500 immune cells or 250 epithelial cells and were excluded from further analysis.

Neutrophil opsonophagocytic killing. Neutrophil killing capacity was evaluated as previously described with minor modification ${ }^{54}$. Briefly, neutrophils were isolated through density-gradient centrifugation, followed by $45 \mathrm{~min}$ incubation with serotype $6 \mathrm{~B}$ pneumococci (inoculation strain, multiplicity of infection 100:1), baby rabbit complement (Mast Group) and human intravenous immunoglobulin (Gamunex; Grifols). In some experiments, recombinant TNF or CXCL10 (Bio-Techne) was added.

Luminex analysis of nasal lining fluid or stimulated nasal cells. Nasal cells collected in RPMI medium containing $1 \%$ penicillin-streptomycin-neomycin (Thermo Fisher Scientific) and 10\% heat-inactivated FBS (Thermo Fisher Scientific) were incubated with $50 \mu \mathrm{g} \mathrm{ml}^{-1}$ DNase I (Sigma Aldrich) at room temperature for $20 \mathrm{~min}$ and filtered over a $70 \mu \mathrm{m}$ filter (Thermo Fisher Scientific). Cells were spun down at $440 \mathrm{~g}$ for $5 \mathrm{~min}$, resuspended, counted and incubated at $250,000 \mathrm{cells} \mathrm{ml}^{-1}$ in 96-well or 384-well plates (Thermo Fisher Scientific). Heat-killed S. pneumoniae inoculation strain was added at a concentration of $5 \mu \mathrm{g} \mathrm{ml}^{-1}$ of total protein (corresponding to $4.3 \times 10^{7} \mathrm{CFU} \mathrm{ml}{ }^{-1}$ ), and cells were incubated for $18 \mathrm{~h}$. Bacterial protein concentration was measured by Bradford assay, using BSA as standard, and titration experiments were performed to determine dose. Supernatant was collected and stored at $-80^{\circ} \mathrm{C}$ until analysis. For nasosorption filters, cytokines were eluted from stored filters using $100 \mu \mathrm{l}$ of assay buffer (Thermo Fisher Scientific) by centrifugation, then the eluate was cleared by further centrifugation at $16,000 \mathrm{~g}$. Prior to analysis, samples were centrifuged for $10 \mathrm{~min}$ at $16,000 \mathrm{~g}$ to clear samples. These were acquired on an LX200 using a 30-plex magnetic human Luminex cytokine kit (Thermo Fisher Scientific) and analyzed with xPonent3.1 software following the manufacturer's instructions. Samples were analyzed in duplicate, and nasosorption samples with a coefficient of variation $(\mathrm{CV})$ of $>25 \%$ were excluded.

RNA extraction and sequencing. Nasal cells were collected in RNALater (Thermo Fisher Scientific) at $-80^{\circ} \mathrm{C}$ until extraction. Extraction was performed using the RNEasy Micro Kit (Qiagen) with on-column DNA digestion. Extracted RNA was quantified using a Qubit (Thermo Fisher Scientific). Sample integrity assessment (Bioanalyzer; Agilent), library preparation and RNA sequencing (Illumina HiSeq4000, 20 million reads, 100 paired-end reads) were performed at the Beijing Genome Institute.

NanoString. Purified blood neutrophils were stored in RLT buffer (Qiagen) with $1 \% 2$-mercaptoethanol (Sigma Aldrich) at $-80^{\circ} \mathrm{C}$ until RNA extraction as above. The single-cell immunology v2 kit (NanoString) was used with 20 pre-amp cycles for all samples. Hybridized samples were prepared on a Prep Station and scanned on a nCounter MAX (NanoString). Raw counts were analyzed using DESeq 2 with internal normalization, which gave lower variance than normalizing to included housekeeping genes. DEGs were identified using a model matrix correcting for repeated individual measurements.

RNA sequencing analysis. Quality control of raw sequencing data was done using FastQC. Mapping to a human reference genome assembly (GRCh38) was done using STAR 2.5.0 ${ }^{55}$. Read counts from the resulting binary alignment map files were obtained with featureCounts using a general transfer format gene annotation from the Ensembl database ${ }^{56,57}$. The R/Bioconductor package DESeq2 was used to identify differentially expressed genes among the samples, after removing absent features (zero counts in more than $75 \%$ of samples) ${ }^{58}$. Genes with a false-discovery rate value of $<0.1$ and an absolute fold change of $>1.5$ were identified as differentially expressed.

Coexpression analysis. For coexpression analysis, counts were normalized using $\log \left[\right.$ counts per million (CPM)], and the $\log _{2}$ [fold change] was calculated for each time point in a subject-wise manner. The coexpression analysis was performed separately for each group (control and LAIV) using the CEMiTool package developed by our group and available at Bioconductor (https://bioconductor.org/packages/ release/bioc/html/CEMiTool.html) ${ }^{39}$. This package unifies the discovery and the analysis of coexpression gene modules, evaluating whether modules contain genes that are over-represented by specific pathways or that are altered in a specific sample group. A $P$-value $=0.05$ was applied for filtering genes with low expression levels.

Statistical analysis. All experiments were performed randomized and blinded. Two-tailed statistical tests were used throughout the study. When log-normalized data were not normally distributed, non-parametric tests were performed and multiple-correction testing (Benjamini-Hochberg) was applied for gene expression and Luminex analysis.

Reporting Summary. Further information on research design is available in the Nature Research Reporting Summary linked to this article.

\section{Data availability}

Raw RNA sequencing data have been deposited in the Gene Expression Omnibus repository, accession number GSE117580. All other underlying data are provided in the manuscript.

\section{References}

51. Gritzfeld, J. F. et al. Experimental human pneumococcal carriage. J. Vis. Exp. 72, e50115 (2013)

52. Jochems, S. P. et al. Novel analysis of immune cells from nasal microbiopsy demonstrates reliable, reproducible data for immune populations, and superior cytokine detection compared to nasal wash. PLoS ONE 12, e0169805 (2017).

53. Gritzfeld, J. F. et al. Density and duration of experimental human pneumococcal carriage. Clin. Microbiol. Infect. 20, O1145-O1151 (2014).

54. Morton, B. et al. Augmented passive immunotherapy with P4 peptide improves phagocyte activity in severe sepsis. Shock 46, 635-641 (2016).

55. Dobin, A. et al. STAR: ultrafast universal RNA-seq aligner. Bioinformatics 29, 15-21 (2013).

56. Liao, Y., Smyth, G. K. \& Shi, W. featureCounts: an efficient general purpose program for assigning sequence reads to genomic features. Bioinformatics $\mathbf{3 0}$, 923-930 (2014)

57. Yates, A. et al. Ensembl 2016. Nucleic Acids Res. 44, D710-D716 (2016)

58. Love, M. I., Huber, W. \& Anders, S. Moderated estimation of fold change and dispersion for RNA-seq data with DESeq2. Genome Biol. 15, 550 (2014). 


\title{
Life Sciences Reporting Summary
}

Nature Research wishes to improve the reproducibility of the work that we publish. This form is intended for publication with all accepted life science papers and provides structure for consistency and transparency in reporting. Every life science submission will use this form; some list items might not apply to an individual manuscript, but all fields must be completed for clarity.

For further information on the points included in this form, see Reporting Life Sciences Research. For further information on Nature Research policies, including our data availability policy, see Authors \& Referees and the Editorial Policy Checklist.

Please do not complete any field with "not applicable" or n/a. Refer to the help text for what text to use if an item is not relevant to your study. For final submission: please carefully check your responses for accuracy; you will not be able to make changes later.

\section{- Experimental design}

1. Sample size

Describe how sample size was determined.

With baseline carriage rates of $50 \%$ (expected based on prior data from our model), 73 participants in each arm were required for $80 \%$ power to detect a $50 \%$ relative increase in pneumococcal acquisition at any time point, after 10\% drop-out.

\section{Data exclusions}

Describe any data exclusions.

Of 130 vaccinated volunteers, five were natural pneumococcal carriers (two in LAIV arm and three in control arm) and were excluded from further analysis. Another 8 subjects in the LAIV arm were excluded following a systematic LAIV dispensing error by a single practitioner, as recommended by the trial steering group. This resulted in a final 55 subjects analysed in the LAIV arm and 62 subjects in the control arm.

\section{Replication}

Describe the measures taken to verify the reproducibility of the experimental findings.

Key findings, including monocyte recruitment to the nasopharynx were validated in an independent patient cohort. All attempts are replication findings were successful.

\section{Randomization}

Describe how samples/organisms/participants were allocated into experimental groups.

\begin{abstract}
Using a permuted-block algorithm (1:1, blocks of 10) held in sealed envelopes, participants were randomised to receive either nasal LAIV (Fluenz Tetra or FluMist Tetra, AstraZeneca, UK, used interchangeably due to procurement shortages) paired with intramuscular placebo $(0.5 \mathrm{ml}$ normal saline), or nasal placebo [control] $(0.2 \mathrm{ml}$ normal saline) paired with intramuscular Quadrivalent Inactivated Influenza Vaccination (Fluarix Tetra, GlaxoSmithKline, UK) (see supplemental methods for flu strains).
\end{abstract}

\section{Blinding}

Describe whether the investigators were blinded to group allocation during data collection and/or analysis.

This was a double-blinded, randomized trial where investigators were blinded during data collection and data analysis 


\title{
6. Statistical parameters
}

For all figures and tables that use statistical methods, confirm that the following items are present in relevant figure legends (or in the Methods section if additional space is needed).

$\mathrm{n} / \mathrm{a} \mid$ Confirmed

\ The exact sample size $(n)$ for each experimental group/condition, given as a discrete number and unit of measurement (animals, litters, cultures, etc.)

$\triangle$ A description of how samples were collected, noting whether measurements were taken from distinct samples or whether the same

sample was measured repeatedly

$\searrow$ A statement indicating how many times each experiment was replicated

The statistical test(s) used and whether they are one- or two-sided

Only common tests should be described solely by name; describe more complex techniques in the Methods section.

A description of any assumptions or corrections, such as an adjustment for multiple comparisons

Test values indicating whether an effect is present

Provide confidence intervals or give results of significance tests (e.g. P values) as exact values whenever appropriate and with effect sizes noted.

A clear description of statistics including central tendency (e.g. median, mean) and variation (e.g. standard deviation, interquartile range)

Clearly defined error bars in all relevant figure captions (with explicit mention of central tendency and variation)

See the web collection on statistics for biologists for further resources and guidance.

\section{- Software}

Policy information about availability of computer code

\section{Software}

Describe the software used to analyze the data in this study.

\author{
STAR-2.5.0a was used to align RNA-Seq read \\ featureCounts v1.5.2 was used to extract numbers of mapped reads from aligned RNAseq \\ data \\ FastQC v0.11.5 was used for RNA-Seq QC analysis \\ MultiQC v1.1 was used for RNA-Seq QC analysis \\ Flowjo v10 was used for analysing flow cytometry data \\ The following R packages were used: \\ DESeq2 v1.14.1 was used to perform differential gene expression analysis from Nanostring \\ and RNA-Seq data \\ Cemitool v0.99.9 was used for co-expression analysis from gene expression data
}

For manuscripts utilizing custom algorithms or software that are central to the paper but not yet described in the published literature, software must be made available to editors and reviewers upon request. We strongly encourage code deposition in a community repository (e.g. GitHub). Nature Methods guidance for providing algorithms and software for publication provides further information on this topic.

\section{- Materials and reagents}

Policy information about availability of materials

\section{Materials availability}

Indicate whether there are restrictions on availability of unique materials or if these materials are only available for distribution by a third party.

\section{Antibodies}

Describe the antibodies used and how they were validated for use in the system under study (i.e. assay and species).
No unique materials were developed or used for this study

Epcam-PE, clone 9C4, Biolegend catalogue \#324206, 1:20 dilution HLADR-PECy7, clone L243, Biolegend catalogue \#307616, 1:20 dilution CD16-APC, clone 3G8, Biolegend catalogue \#302012, 1:20 dilution CD66b-FITC, clone G10F5, Biolegend catalogue \#305104, 1:20 dilution CD3-APCH7, clone SK7, BD catalogue \#560176, 1:33 dilution CD14-PercpCy5.5, clone M\$P9, BD catalogue \#562692, 1:20 dilution CD45-PACOrange, clone HI30, ThermoFisher catalogue \#MHCD4530, 1:20 dilution TIGIT-PECy7, clone A15153G, Biolegend catalogue \#372714, 1:20 dilution

We previously reported the use of this panel on nasal cells. (Jochems et al, Plos One, 2017, https://doi.org/10.1371/journal.pone.0169805). Fluorescent minus one controls were used to validate the panel.

Utilized lot IDs were not recorded for this study. 


\section{Eukaryotic cell lines}

a. State the source of each eukaryotic cell line used.

b. Describe the method of cell line authentication used.

c. Report whether the cell lines were tested for mycoplasma contamination.

d. If any of the cell lines used are listed in the database of commonly misidentified cell lines maintained by ICLAC, provide a scientific rationale for their use. no eukaryotic cell lines were used

no eukaryotic cell lines were used

no eukaryotic cell lines were used

no eukaryotic cell lines were used

\section{- Animals and human research participants}

Policy information about studies involving animals; when reporting animal research, follow the ARRIVE guidelines

\section{Description of research animals}

Provide all relevant details on animals and/or

No animals were used

animal-derived materials used in the study.

Policy information about studies involving human research participants

\section{Description of human research participants}

Describe the covariate-relevant population characteristics of the human research participants.
Age (median + range) $=20(18-48)$;

Female gender no $(\%)=79(58.9)$;

Mean pneumococcus inoculation dose in CFU (sd) = 75699 (8478);

The volunteers were healthy young adults 